\title{
Pilot Study to Evaluate Serum Soluble Mesothelin-Related Peptide (SMRP) as Marker for Clinical Monitoring of Pleural Mesothelioma (PM): Correlation with Modified RECIST Score
}

\author{
Federica Grosso ${ }^{1,2, *}$, Matilde Mannucci ${ }^{3} \mathbb{D}$, Francesca Ugo ${ }^{4}$, Paola Ferro ${ }^{5}$, Maurizio Cassinari ${ }^{6}$, \\ Antonella Vigani ${ }^{7}$, Antonina Maria De Angelis ${ }^{1}$, Sara Delfanti ${ }^{1}$, Michela Lia ${ }^{1}$, Roberto Guaschino ${ }^{6}$, \\ Stefano Barbero ${ }^{8}$, Silvio Roncella ${ }^{5}$, Ugo Giannoni ${ }^{9}$, Marinella Bertolotti ${ }^{4}$, Maria Pia Pistillo ${ }^{10}$ \\ and Vincenzo Fontana ${ }^{3}$
}

Citation: Grosso, F.; Mannucci, M.; Ugo, F.; Ferro, P.; Cassinari, M.;

Vigani, A.; De Angelis, A.M.; Delfanti, S.; Lia, M.; Guaschino, R.; et al. Pilot Study to Evaluate Serum Soluble Mesothelin-Related Peptide (SMRP) as Marker for Clinical Monitoring of Pleural Mesothelioma (PM): Correlation with Modified RECIST Score. Diagnostics 2021, 11, 2015. https://doi.org/10.3390/ diagnostics11112015

Academic Editors: Luca Falzone and Chung-Che (Jeff) Chang

Received: 6 August 2021

Accepted: 28 October 2021

Published: 29 October 2021

Publisher's Note: MDPI stays neutral with regard to jurisdictional claims in published maps and institutional affiliations.

Copyright: (c) 2021 by the authors. Licensee MDPI, Basel, Switzerland. This article is an open access article distributed under the terms and conditions of the Creative Commons Attribution (CC BY) license (https:// creativecommons.org/licenses/by/ $4.0 /)$.
1 Mesothelioma Unit, Azienda Ospedaliera SS Antonio e Biagio e Cesare Arrigo, 15121 Alessandria, Italy; antonina.deangelis@ospedale.al.it (A.M.D.A.); sara.delfanti@ospedale.al.it (S.D.); michela.lia@ospedale.al.it (M.L.)

2 Translational Medicine, Dipartimento Attività Integrate Ricerca e Innovazione, Azienda Ospedaliera SS Antonio e Biagio e Cesare Arrigo, 15121 Alessandria, Italy

3 Clinical Epidemiology Unit, IRCCS Ospedale Policlinico San Martino, 16132 Genova, Italy; matilde.mannucci@hsanmartino.it (M.M.); vincenzo.fontana@hsanmartino.it (V.F.)

4 Infrastruttura Ricerca Formazione e Innovazione, Dipartimento Attività Integrate Ricerca e Innovazione, Azienda Ospedaliera SS Antonio e Biagio e Cesare Arrigo, 15121 Alessandria, Italy; fugo@ospedale.al.it (F.U.); MBertolotti@ospedale.al.it (M.B.)

5 Histopathology and Cytopathology Unit, Azienda Sanitaria Locale 5, 19121 La Spezia, Italy; paola.ferro@asl5.liguria.it (P.F.); roncy88@alice.it (S.R.)

6 Laboratory Unit, Azienda Ospedaliera SS Antonio e Biagio e Cesare Arrigo, 15121 Alessandria, Italy; mcassinari@ospedale.al.it (M.C.); rguaschino@ospedale.al.it (R.G.)

7 Oncology Division, Azienda Sanitaria Locale 5, 19121 La Spezia, Italy; antonella.vigani@asl5.liguria.it

8 Radiology Department, Azienda Ospedaliera SS Antonio e Biagio e Cesare Arrigo, 15121 Alessandria, Italy; stefano.barbero@ospedale.al.it

9 Radiodiagnostic Division, Azienda Sanitaria Locale 5, 19121 La Spezia, Italy; ugo.giannoni@asl5.liguria.it

10 Tumor Epigenetics Unit, IRCCS Ospedale Policlinico San Martino, 16132 Genova, Italy; mariapia.pistillo@gmail.com

* Correspondence: federica.grosso@ospedale.al.it

Abstract: A soluble mesothelin-related peptide (SMRP) is the only FDA-approved biomarker for diagnosis of pleural mesothelioma (PM) and the most used for monitoring treatment. Radiological assessment of PM, based on modified RECIST (mRECIST) criteria, is challenging. This pilot study was designed to evaluate whether SMRP levels correlated over time with mRECIST score. Serial serum samples from PM patients were collected and SMRP levels were measured and compared with the mRECIST score obtained through centralized CT scans by blinded review. The withinpatient SMRP-mRECIST relationship over time was estimated through a normal random-effects regression approach applied to the log-transformed mRECIST score. Overall, 58 PM patients were included (46 males and 12 females) with a median age at diagnosis of 67 years ( $\min -\max =48-79)$, 44 (76\%) with epithelioid and 14 (24\%) with non-epithelioid histology. The total number of SMRP measurements and CT scans considered for analysis was 183. There was a statistically significant correlation between SMRP and mRECIST score in the 2 cohorts considered both separately and jointly. These results, although exploratory, suggest that SMRP measurement might be considered as an adjunct to monitor PM patients in order to delay CT scans time interval, thus warranting further investigation.

Keywords: mesothelioma; mesothelin; serum biomarkers; modified RECIST; longitudinal study 


\section{Introduction}

Pleural mesothelioma (PM) is a rare cancer of the mesothelial surfaces with dismal prognosis. PM patients have a median overall survival (OS) ranging from 4 to 13 months for untreated patients and from 6 to 18 months for treated patients [1,2]. Since 2004, platinum and antifolates have been the gold standard for the first line treatment of patients with advanced disease with a median OS of 12 months [3]. Recently, in the phase III pivotal trial CheckMate 743, the combination of nivolumab plus ipilimumab significantly prolonged OS with respect to standard chemotherapy [4]. Although there is no approved second line, the combination of ramucirumab and gemcitabine showed significant OS improvements over gemcitabine in the phase II RAMES study and nivolumab in the salvage setting remarkably improved progression-free and OS in the phase III placebo-controlled CONFIRM trial [5-7].

Despite the recent progress, there is still an urgent need for active therapies in this disease and for tools that could assist clinicians in improving the management of patients.

One of the most controversial issues, due to the unique morphology of tumor growth, is the evaluation of the radiological response to treatment not only in the context of clinical research but also in everyday practice. Specific response criteria, the so-called modified RECIST (mRECIST) criteria for PM, have been developed in 2004 and recently updated to help clinicians standardize the measurement of lesions thus allowing a better and more reproducible classification of response. The application of mRECIST criteria, however, requires expert and trained radiologists [8,9], and although the criteria are most often used in clinical trials, their use in clinical practice is still limited.

Serum biomarkers mirroring disease extension, in addition to the use of specific radiological criteria, would be of significant value and could represent an attractive option to optimize the clinical management of PM patients.

Among biomarkers, the most investigated is mesothelin, a membrane-bound glycoprotein, previously proposed for diagnostic use, produced at low levels by normal mesothelial cells and overexpressed in certain tumors including PM, pancreatic adenocarcinoma, ovarian, and lung cancer [10-12]. Serum soluble mesothelin-related peptide (SMRP) is the form of mesothelin released by PM cells into the blood where it can be detected and measured. Although subsequent evidence has indicated poor performance of SMRP as a screening test due to low sensitivity, it remains the most used and the only FDA-approved serum biomarker for diagnosis [13].

SMRP can be useful for the monitoring of therapy. Many studies have suggested a correlation between SMRP levels and PM response to systemic therapy [14-17]. A systematic review including 20 studies indicated that the decrease in serum levels of SMRP might correlate with response to treatment [18]. Moreover, in a small series of 10 PM patients followed at a single Italian institution, a longitudinal statistical analysis indicated that SMRP levels and pleural thickness showed very similar trends confirming a previously reported observation of a strong correlation between the two clinical markers [19].

This exploratory, descriptive pilot study was directed towards assessing the correlation between SMRP levels and disease extension, as described by the mRECIST criteria, during the patients' clinical course. To this end, we used, for the first time, a statistical method able to estimate patient-specific rates of change in both clinical markers by analyzing the longitudinal profile of repeated measurements of the same markers.

\section{Materials and Methods}

\subsection{Patients}

This is a retrospective, observational pilot study designed to estimate the correlation over time between SMRP levels and mRECIST evaluations, independently of the anticancer treatment. The study population consisted of two different PM patients' cohorts, the Alessandria (AL) cohort and the La Spezia (SP) cohort, including 40 and 18 patients, respectively. The AL cohort, followed by the Mesothelioma Unit at Azienda Ospedaliera SS Antonio e Biagio e Cesare Arrigo, Alessandria (Italy), was recruited from June 2014 to April 2020 and the follow-up period ended by April 2020. The SP cohort followed by 
the Oncology Unit at the Azienda Sanitaria Locale 5, La Spezia (Italy), was recruited from March 2010 to May 2015 and the follow-up period ended by September 2017. In both cohorts, the diagnosis was based on histological examination of tumor samples obtained by means of thoracoscopy or computed tomography (CT) guided biopsy and analyzed through the appropriate immunohistochemistry according to international reference guidelines [20].

In both cohorts, CT scans and peripheral blood examinations, inclusive of SMRP, were performed according to routine clinical practice. For each patient, we considered all CT scans performed along with the whole clinical history. In addition, we selected the SMRP measurement closest to the CT scans. The CT scans considered for analysis were stored in the AL and SP hospitals' radiological archives. All the CT scans were retrieved, anonymized and sent for centralized review performed by the same expert radiologist, at the Azienda Ospedaliera SS Antonio e Biagio e Cesare Arrigo, Alessandria, Italy, blind to clinical data and SMRP levels. The disease extension was described through a numerical score measured according to the mRECIST criteria and referred to as the mRECIST score.

Patients' characteristics, systemic treatments and CT scan reports, including the description of tumor extension and response to therapy, were collected in two separate databases at each participating center (AL and SP). A common database, including patients' data from both cohorts, SMRP measurements and the mRECIST score over time was generated for the purpose of this study.

\subsection{Measurement of Serum SMRP}

Serum concentrations of SMRP were measured using the chemiluminescent enzyme immunoassay (CLEIA) kit (Lumipulse G Mesothelin, Fujirebio Europe, Ghent, Belgium) for the AL samples and the enzyme-linked immunosorbent assay (ELISA) kit (MesoMark, Fujirebio, Japan) for the SP samples, according to the respective manufacturers' instructions.

Aliquots of each serum sample were centrifuged $\left(1500 \times \mathrm{g}\right.$ for $10 \mathrm{~min}$ at $\left.4{ }^{\circ} \mathrm{C}\right)$ and supernatants were stored at $-20^{\circ} \mathrm{C}$ until assays were performed. Each sample was tested in duplicate with deviation between duplicates lower than $10 \%$. The lowest sensitivity thresholds were $\leq 0.1 \mathrm{nmol} / \mathrm{L}$ for the CLEIA kit and $0.3 \mathrm{nmol} / \mathrm{L}$ for the ELISA kit, respectively.

Results were expressed as the geometric mean of SMRP concentrations.

\subsection{CT Imaging and Modified RECIST Score}

All patients had CT scans according to routine practice. Patients who received chemotherapy had a CT scan every 3-4 months, whereas patients in the follow-up phase, or on treatment holidays, or candidates for best supportive care only, had a CT scan at clinically appropriate intervals (i.e., every 6-8 months) according to internal guidelines. In this framework, the pleural rind was measured through the sum of six perpendicular chest wall measurements from three separate sections of the pleural tumor, preferably above the carina. Tumor thickness perpendicular to the chest wall or mediastinum was measured in two positions at three separate levels on transverse CT slices; levels considered for measurements required to be at least $1 \mathrm{~cm}$ apart and related to anatomical landmarks to allow reproducible assessment at later time points. The sum of the six measurements defined a pleural unidimensional measure. Bidimensionally measurable lesions, such as mediastinal lymph nodes, were recorded unidimensionally, as for RECIST 1.1, and were added to the pleural measurement. Criteria for partial response and progressive disease were the same as in the original RECIST 1.1. system [9].

\subsection{Statistical Analysis}

Descriptive statistics were applied to explore patient and disease characteristics in the two cohorts separately and jointly. Categorical variables (gender, histology, ECOG-PS) were expressed as absolute and relative frequencies (percentages), whereas continuous variables (age at diagnosis, months from diagnosis and years of follow-up) were summarized using median value and inter-quartile range (IQR). The distributions of SMRP levels and 
mRECIST score were described using the geometric mean (GM) and corresponding 95\% confidence limits (95\% CL). Analysis of contingency tables and related chi-square test were performed to assess the correlation between categorical variables, whereas the Student's $t$-test was applied to compare distributions of continuous variables among subgroups of patients.

The SMRP-mRECIST relationship over time was evaluated through a normal randomeffects regression approach [21] applied to the log-transformed mRECIST (log-n-mRECIST) score. Such a statistical methodology is appropriate to manage two important data features: firstly, the random-effects regression allows consideration of the longitudinal structure of within-patient repeated measurements; secondly, the log-transformation is useful to fulfill the normality assumption, to reduce any undue influence on the regression results of aberrant data points and, at the same time, to estimate the geometric mean ratio (GMR), along with corresponding 95\% CL, as an index of SMRP-mRECIST association over the follow-up period.

All analyses were performed using Stata (StataCorp. Stata Statistical Software. Release 16. College Station, TX, USA, 2020).

\section{Results}

\subsection{Patients' Characteristics}

The study sample included 58 PM patients ( 40 from AL and 18 from SP) diagnosed in the period 2010-2017 and characterized by an individual profile composed of age at diagnosis, months from diagnosis to the first SMRP measurement, years of follow-up, gender, histology, Eastern Cooperative Oncology Group performance status (ECOG-PS), and vital status at the end of follow-up and treatment (Table 1). Each patient was followed from the date of first SMRP measurement until the date of death or last follow-up visit. Table 1 summarizes patients' baseline characteristics, split in the two cohorts and analyzed together. In the whole population, median age was 67 years (IQR $=62-72), 46(79.3 \%)$ were males and $12(20.7 \%)$ females, histology was epithelioid in $44(75.9 \%)$ patients and non-epithelioid in 14 (24.1\%). The median time from diagnosis to the first determination of SMRP was 2.1 months (IQR $=0.9-12.2$ ). Patients' ECOG PS was $\leq 1$ in $35(60.3 \%)$ and $>1$ in $23(37.7 \%)$. The median length of follow-up was 1.8 years (IQR $=1.0-2.7)$ and at the end of the study period only six patients $(10.3 \%)$ were still alive.

Table 1. Patients' characteristics in MPM cohorts.

\begin{tabular}{|c|c|c|c|c|c|c|c|c|c|c|c|c|}
\hline \multirow{2}{*}{ Variable } & \multicolumn{4}{|c|}{ AL Cohort } & \multicolumn{4}{|c|}{ SP Cohort } & \multicolumn{4}{|c|}{ Both Cohorts } \\
\hline & No. & $\%$ & P50 & IQR & No. & $\%$ & P50 & IQR & No. & $\%$ & P50 & IQR \\
\hline Age at diagnosis & & & 67 & $61-72$ & & & 68 & $63-72$ & & & 67 & $62-72$ \\
\hline Days from diagnosis & & & 5 & $3-27$ & & & 3 & $0-56$ & & & 5 & $2-30$ \\
\hline Years of follow-up & & & 1.4 & $0.9-2.4$ & & & 2.5 & $1.7-3.1$ & & & 1.8 & $1.0-2.7$ \\
\hline $\begin{array}{c}\text { Mortality } \\
\text { rate } / 1000 / \text { month }^{1}\end{array}$ & & & 41.8 & $29.8-58.4$ & & & 33.8 & $21.3-53.6$ & & & 38.6 & $29.4-50.7$ \\
\hline \multicolumn{13}{|l|}{ Gender } \\
\hline Male & 29 & 72.5 & & & 17 & 94.4 & & & 46 & 79.3 & & \\
\hline Female & 11 & 27.8 & & & 1 & 5.6 & & & 12 & 20.7 & & \\
\hline \multicolumn{13}{|l|}{ Histology } \\
\hline Epithelioid & 30 & 66.7 & & & 14 & 77.8 & & & 44 & 75.9 & & \\
\hline Non-epithelioid & 10 & 33.3 & & & 4 & 22.2 & & & 14 & 24.1 & & \\
\hline \multicolumn{13}{|l|}{ ECOG-PS } \\
\hline$\leq 1$ & 28 & 70.0 & & & 7 & 38.9 & & & 35 & 60.3 & & \\
\hline$>1$ & 12 & 30.0 & & & 11 & 61.1 & & & 23 & 39.7 & & \\
\hline \multicolumn{13}{|l|}{ Vital status } \\
\hline Alive & 6 & 15.0 & & & 0 & 0.0 & & & 6 & 10.3 & & \\
\hline Dead & 34 & 85.0 & & & 18 & 100.0 & & & 52 & 89.7 & & \\
\hline \multicolumn{13}{|l|}{ Therapy } \\
\hline Chemotherapy & 6 & 15.0 & & & 15 & 83.3 & & & 21 & 36.2 & & \\
\hline $\begin{array}{l}\text { Chemo and } \\
\text { immunotherapy }\end{array}$ & 13 & 32.5 & & & 0 & 0.0 & & & 13 & 22.4 & & \\
\hline $\begin{array}{l}\text { Chemo and } \\
\text { antiangiogenic }\end{array}$ & 21 & 52.5 & & & 0 & 0.0 & & & 21 & 36.2 & & \\
\hline Supportive care & 0 & 0.0 & & & 3 & 16.6 & & & 3 & 5.2 & & \\
\hline Whole sample & 40 & 100.0 & - & - & 18 & 100.0 & - & - & 58 & 100.0 & - & - \\
\hline
\end{tabular}

AL: Alessandria; SP: La Spezia; P50: median value; IQR: inter-quartile range. ${ }^{1}$ Mean rate and corresponding $95 \%$ confidence interval. 
Regarding treatment, in the AL cohort 6 (15.0\%) patients received standard chemotherapy, 13 (32.5\%) chemotherapy and immunotherapy, 21 (52.5\%) chemotherapy and antiangiogenics. In the SP cohort $15(83.3 \%)$ patients received chemotherapy and $3(16.6 \%)$ best supportive care only.

At baseline, some differences between the statistical profiles of the two cohorts were highlighted (Table 1). AL patients had better daily living performance (ECOG-PS $\leq 1: 70.0 \%$ vs. $38.9 \%$ ) and the SP cohort showed a median follow-up period which was approximately twice (2.5 years; IQR = 1.7-3.1) that observed in the AL cohort (1.4 years; IQR = 0.9-2.4). In addition, a death risk excess of approximately $25 \%$ was found in the AL patients (rate $=41.8$ per 1000 per month; 95\% CL $=29.8-58.4$ ) when compared to the SP patients (rate $=33.8$ per 1000 per month; $95 \%$ CL $=21.3-53.6$ ) (Table 1 ).

\subsection{SMRP and mRECIST Measurements}

Over the follow-up period, AL and SP patients had 141 and 42 mRECIST measurements, respectively, corresponding to an annual mean frequency of 2.1 and 1.0 per patient. Consistently, these measurements were selected for the analysis using the time closeness to each CT scan examination as a selection criterion for the SMRP measurements. Using this framework, the median time between each CT scan examination and the corresponding SMRP measurement was 7 days (IQR = 4-13) for AL patients and 30 days $(I Q R=16-50)$ for SP patients.

Table 2 shows the distributions of SMRP levels and mRECIST score values at baseline by cohort and histological subtype. In both cohorts, the GM of SMRP levels was $2.77 \mathrm{nM}$ (95\% CL $=2.11-3.66)$ in epithelioid patients and $1.89 \mathrm{nM}(95 \% \mathrm{CL}=1.16-3.09)$ in nonepithelioid patients. GM levels of SMRP were slightly lower in AL patients (2.42 nM, $95 \% \mathrm{CL}=1.74-3.36$ vs. $2.81 \mathrm{nM}, 95 \% \mathrm{CL}=2.03-3.88)$; the imbalance was mainly due to differences between non-epithelioid PM patients $(1.43 \mathrm{nM}, 95 \% \mathrm{CL}=0.76-7.70 \mathrm{vs}$. $3.81 \mathrm{nM}$, $95 \% \mathrm{CL}=1.92-7.56)$. Similar results were obtained for the mRECIST distribution. AL patients scored on average lower $(\mathrm{GM}=82.5,95 \% \mathrm{CL}=69.5-97$ vs. $\mathrm{GM}=94.4,95 \% \mathrm{CL}=$ 77.0-115.6), although in this case much of the observed divergence was observed within the epithelioid PM subgroup $(\mathrm{GM}=81.8,95 \% \mathrm{CL}=67.0-99.8$ vs. $\mathrm{GM}=97.1,95 \% \mathrm{CL}=$ 76.6-123.0).

Table 2. Determinations of SMRP levels and mRECIST score in all MPM patients and by histological subtypes.

\begin{tabular}{cccccccc}
\hline \multirow{2}{*}{ Clinical Marker } & \multirow{2}{*}{ Histology } & \multicolumn{2}{c}{ AL Cohort } & \multicolumn{2}{c}{ SP Cohort } & \multicolumn{2}{c}{ Both Cohorts } \\
\cline { 2 - 7 } & & GM & $\mathbf{9 5 \%}$ CL & GM & 95\% CL & GM & 95\% CL \\
\hline \multirow{3}{*}{ SMRP levels (nM) } & Epithelioid & 2.88 & $1.99-4.16$ & 2.57 & $1.78-3.71$ & 2.77 & $2.11-3.66$ \\
& Non-epithelioid & 1.43 & $0.76-7.70$ & 3.81 & $1.92-7.56$ & 1.89 & $1.16-3.09$ \\
\cline { 2 - 7 } & Whole sample & 2.42 & $1.74-3.36$ & 2.81 & $2.03-3.88$ & 2.53 & $1.98-3.23$ \\
\hline \multirow{2}{*}{ mRECIST score } & Epithelioid & 81.8 & $67.0-99.8$ & 97.1 & $76.6-123.0$ & 86.3 & $74.2-100.5$ \\
& Non-epithelioid & 84.7 & $59.9-119.7$ & 85.5 & $55.0-133.1$ & 84.9 & $64.9-111.2$ \\
\cline { 2 - 7 } & Whole sample & 82.5 & $69.5-97.8$ & 94.4 & $77.0-115.6$ & 86.0 & $75.4-98.0$ \\
\hline
\end{tabular}

SMRP: soluble mesothelin peptide; mRECIST: modified RECIST; GM: geometric mean; 95\% CL: 95\% confidence limits for GM.

\subsection{Relationship between SMPR and mRECIST}

The results of the random-effects regression analysis are shown in Table 3. The SMRPmRECIST association was evaluated in two different ways. Firstly, SMRP entered the regression equation as a four-category variable derived by dividing the biomarker levels according to the quartiles of the original measurements. Secondly, SMPR was used as a base-2-log-transformed continuous variable (log-2-SMRP). In both cases, a clear upward trend in mRECIST score was estimated as a function of increasing SMRP levels. Specifically, using the first SMRP category as a reference, mRECIST GM values showed a positive change of approximately $15-20 \%$ on average as SMRP moved from one category to the next 
highest. This result was consistent with that obtained by replacing the categorical terms of SMRP with log-2-SMRP. In this case, it was observed that when the SMRP levels doubled (i.e., increased by $100 \%$ ) the proportional change in mRECIST score was approximately $+16 \%$ in the AL cohort $(\mathrm{GMR}=1.16,95 \% \mathrm{CL}=1.11-1.22 ; p$-value $<0.001)$ and $+18 \%$ in the $\mathrm{SP}$ cohort $(\mathrm{GMR}=1.18,95 \% \mathrm{CL}=1.06-1.31 ; p$-value $<0.003)$ and, as a consequence, a similar result was obtained for the joint-cohort analysis (GMR $=1.19,95 \% \mathrm{CL}=1.13-1.25$; $p$-value $<0.001)$. It is worth noting that overlapping results were also computed for the two histological subtypes (epithelioid: $\mathrm{GMR}=1.20,95 \% \mathrm{CL}=1.13-1.27$; non-epithelioid: $\mathrm{GMR}=1.17,95 \% \mathrm{CL}=1.10-1.25)$.

Table 3. Association between SMRP levels and mRECIST score estimated through a log-normal random-effects regression method in MPM cohorts.

\begin{tabular}{|c|c|c|c|c|c|c|c|c|c|c|c|c|c|c|}
\hline \multicolumn{5}{|c|}{ AL Cohort } & \multicolumn{5}{|c|}{ SP Cohort } & \multicolumn{5}{|c|}{ Both Cohorts } \\
\hline SMRP (nM) & GM & GMR & $95 \%-C L$ & $\begin{array}{c}p- \\
\text { Value }\end{array}$ & SMRP (nM) & GM & GMR & 95\%-CL & $\begin{array}{c}p- \\
\text { Value }\end{array}$ & SMRP (nM) & GM & GMR & 95\%-CL & $\begin{array}{c}p- \\
\text { Value }\end{array}$ \\
\hline $\begin{array}{l}\text { Categories } \\
\text { (median) }\end{array}$ & & & & 0.010 & $\begin{array}{l}\text { Categories } \\
\text { (median) }\end{array}$ & & & & 0.043 & $\begin{array}{l}\text { Categories } \\
\text { (median) }\end{array}$ & & & & 0.006 \\
\hline $\begin{array}{c}0.14-0.99 \\
(0.53)\end{array}$ & 61.1 & 1.00 & (Ref.) & & $\begin{array}{c}1.10-1.99 \\
(1.61)\end{array}$ & 95.3 & 1.00 & (Ref.) & & $\begin{array}{c}0.14-1.14 \\
(0.61)\end{array}$ & 73.3 & 1.00 & (Ref.) & \\
\hline $\begin{array}{c}1.00-1.71 \\
(1.39)\end{array}$ & 68.8 & 1.13 & $0.97-1.31$ & & $\begin{array}{c}2.00-2.78 \\
(2.42)\end{array}$ & 104.4 & 1.09 & $0.83-1.44$ & & $\begin{array}{c}1.15-2.01 \\
(1.57)\end{array}$ & 77.2 & 1.05 & $0.90-1.24$ & \\
\hline $\begin{array}{c}1.72-3.57 \\
(2.55)\end{array}$ & 77.2 & 1.26 & $1.06-1.51$ & & $\begin{array}{c}2.79-5.72 \\
(3.65)\end{array}$ & 123.6 & 1.29 & $1.00-1.69$ & & $\begin{array}{l}2.02-3.94 \\
(2.79)\end{array}$ & 90.2 & 1.23 & $1.02-1.49$ & \\
\hline $\begin{array}{c}3.58-63.9 \\
(7.15)\end{array}$ & 84.4 & 1.38 & $1.14-1.67$ & & $\begin{array}{c}5.73-12.3 \\
(9.10)\end{array}$ & 134.5 & 1.41 & $1.06-1.88$ & & $\begin{array}{c}3.95-63.9 \\
(7.15)\end{array}$ & 99.7 & 1.36 & $1.13-1.64$ & \\
\hline Log-2-levels & & & & $<0.001$ & Log-2-levels & & & & 0.003 & Log-2-levels & & & & $<0.001$ \\
\hline Linear trend & & 1.16 & $1.11-1.22$ & & Linear trend & & 1.18 & $1.06-1.31$ & & Linear trend & & 1.19 & $1.13-1.25$ & \\
\hline
\end{tabular}

SMRP: soluble mesothelin peptide; mRECIST: modified RECIST; GM: mRECIST geometric mean; GMR: mRECISTgeometric mean ratio, i.e., ratio between the mRECIST GM in each SMRP category and MRECIST GM in the first SMRP category; Ref.: SMRP reference category; median: SMRP median value in each SMRP category; 95\%-CL: 95\% confidence limits for GMR; $p$-value: probability level associated with the likelihood ratio test; Log-2-levels: base-2 log-transformedSMRP levels.

Figure 1 depicts the estimated association in terms of trajectories in the log-n-mRECIST score over log-2-SMRP levels in each patient (thin lines) and averaged across all patients (thick lines) in both cohorts separately and jointly.

Figure 2 shows two examples of consistency between SMRP level decrease (Figure 2A) or increase (Figure 2B) and mRECIST measurements/disease extension (Figure 2A: disease treatment response; Figure 2B: disease progression). These examples are distinguished by their subheadings. They provide a concise and precise description of the experimental results, their interpretation, as well as the experimental conclusions that can be drawn. 

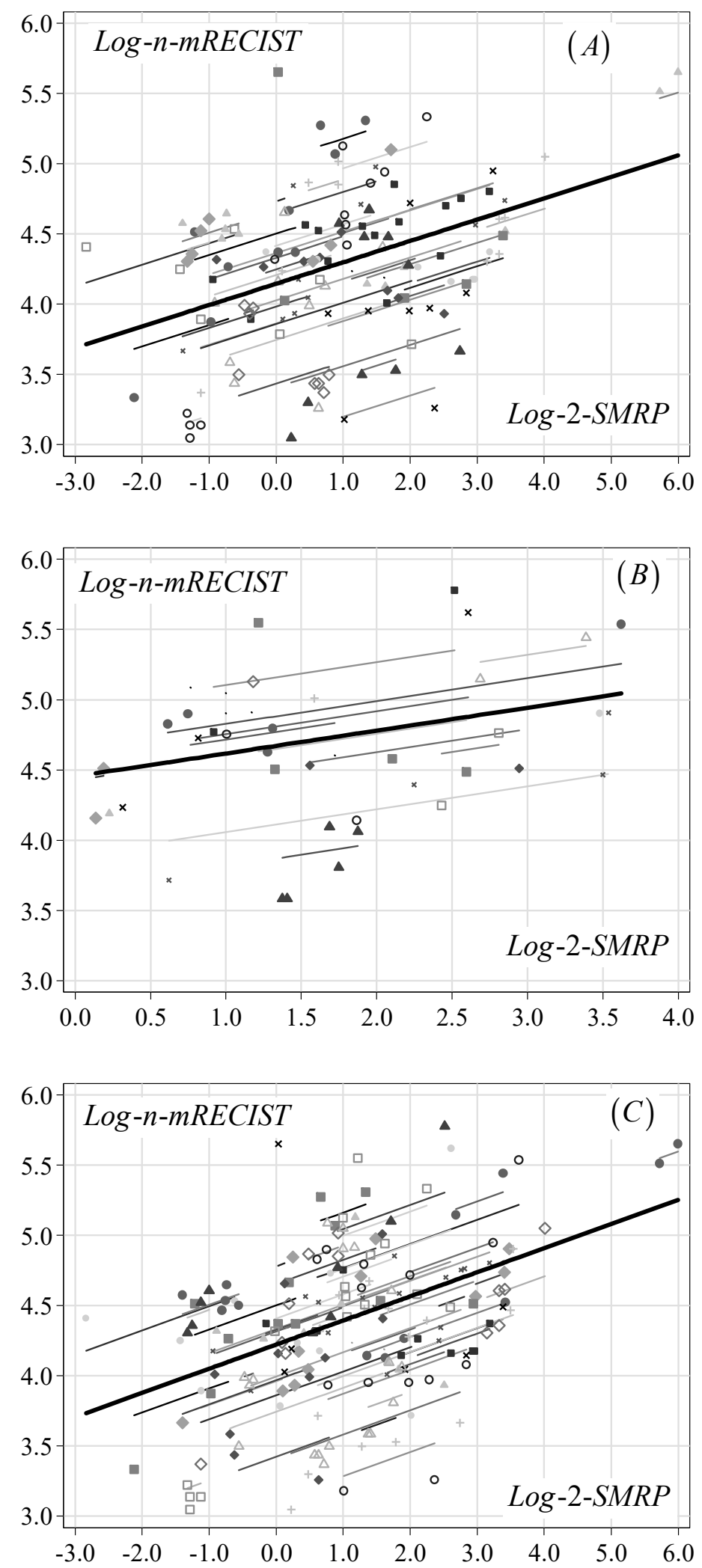

Figure 1. Association between mRECIST score and SMRP levels (double logarithmic scale) estimated through a normal random-effects regression in the AL cohort (A), SP cohort (B) and both cohorts (C). Note: mRECIST: modified RECIST; SMRP: soluble mesothelin-related peptides; log-n: natural logarithm; log-2: base-2 logarithm. Symbols: observed patient-specific levels of log-n-mRECIST score and $\log$-2-SMRP. Thin lines: patient-specific linear relationships between log-n-mRECIST score and log-2-SMRP levels. Thick lines: overall relationship obtained as an average across all patients. 
A

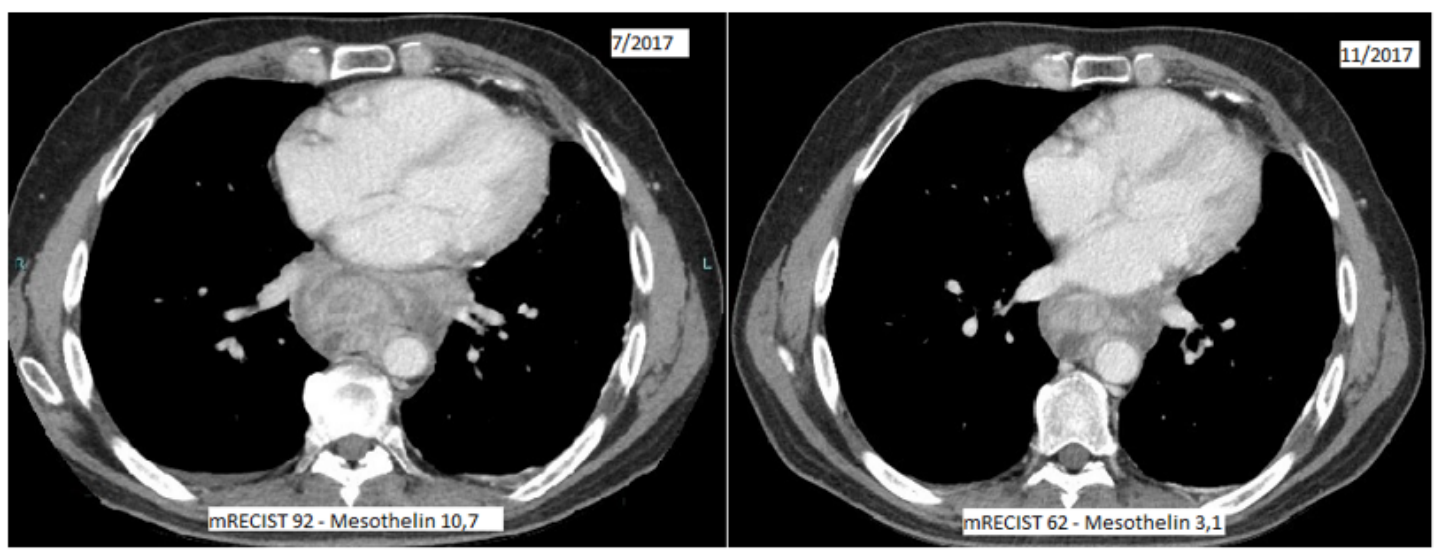

B
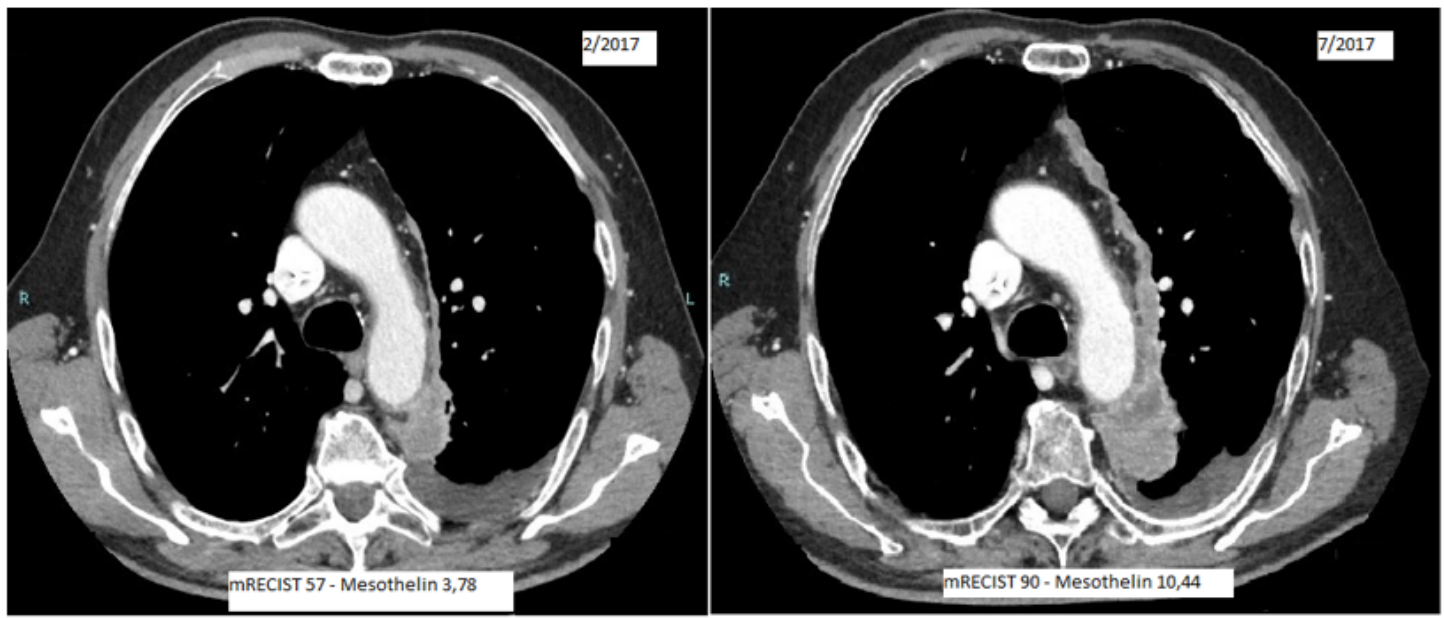

Figure 2. Examples of correlation between mRECIST score and SMRP levels. (A) mRECIST reduction (disease treatment response) correlates with SMRP reduction; (B) mRECIST increase (disease progression) correlates with SMRP increase; mRECIST: modified RECIST; SMRP: soluble mesothelin-related peptides.

\section{Discussion}

This exploratory pilot study was performed to assess the correlation between time changes in SMRP levels and in disease extension, as described by the mRECIST scores, in a sample of 58 PM patients followed at two hospitals in the north of Italy, both located in areas (Alessandria and La Spezia Provinces) with a high incidence of PM. It was undertaken to confirm a previous observation, made in a smaller series of PM patients, that SMRP levels and pleural thickness had very similar trends [19]. On this occasion, we chose the mRECIST score instead of the pleural thickness because it was the standardized way to describe disease extension and represented the basis of the criteria for response evaluation. We decided to analyze two different cohorts of patients together to increase the number of subjects and therefore the statistical power, but also to evaluate the association in two different circumstances.

In our study, the within-patient correlation over time between SMRP level and mRECIST changes was evaluated independently of systemic treatment or follow-up phase.

To determine the correlation, we used a normal random-effects regression approach [21] applied to the log-transformed mRECIST. Such a statistical tool has the clear advantage over other regression methods of indicating the joint time trajectory of both clinical markers within each patient. In this way, positive or negative changes in both markers can be mainly attributed to the corresponding positive or negative changes in disease extension given 
that all comparisons were based on longitudinal repeated measurements undertaken on the same patient. The final result was represented by a single index of association (GMR) derived as an average computed over all patients which summarizes the joint marker behavior during the whole follow-up period. To our knowledge, such a statistical approach has not yet been applied in a PM clinical setting to evaluate the direction and intensity of association between the two markers.

Our results showed a statistically significant correlation between SMRP and mRECIST score in the two cohorts when analyzed both separately and jointly. An mRECIST score mean increase of approximately $20 \%$ was estimated for each doubling in SMRP levels when the data were considered together. Similar results were also obtained for the two histology sub-types (epithelioid: $+20 \%$; non-epithelioid: $+17 \%$ ).

Although the two cohorts differed in some baseline characteristics, in particular oncological treatments, length of follow-up and method used to measure SMRP levels (CLEIA in the AL cohort and ELISA in the SP cohort), all the comparisons were performed within patients, namely by taking into account all patient-specific features, thus providing a more accurate estimation of the SMRP-mRECIST relationship over time. The small sample size and the lack of a specific samples collection timeline do though represent a weakness of the present study, which was aimed at producing a proof of concept for the use of SMRP as a marker for the clinical monitoring of PM.

Blood biomarkers whose levels reflect disease extension are very useful in the clinical management of cancer patients and the availability of a reliable biomarker for PM patients is of great importance, since these patients are currently monitored only with radiological examinations that are often very difficult to interpret. SMRP has been demonstrated to be a useful biomarker for the monitoring of treatment. In a surgical series of 102 patients, SMRP level decreased immediately after surgery and increased over time during disease progression in $82.4 \%$ of patients with progressive disease suggesting that it is a promising serum biomarker for the detection of recurrence after resection that may have value in clinical practice [14]. In a series of 40 patients with SMRP $>1 \mathrm{nM}$ and receiving chemotherapy and immunotherapy, an increase in serial measurements of $10 \%$ or greater correlated with a probability of $75 \%$ of radiological progression using mRECIST criteria and with worse OS [22]. In a series of $41 \mathrm{PM}$ patients receiving chemotherapy or best supportive care, a $10 \%$ rise in SMRP could predict radiological progression with a sensitivity of $96 \%$ and a specificity of $74 \%$ [23]. Other studies have confirmed the value of SMRP for the monitoring of therapy; a systematic review including 20 studies (18 prospective and 2 retrospective) for a total of 1578 patients suggested that SMRP measured before and after treatment can track treatment response as seen on serial CT scans [18].

A blood biomarker that could have a role in addition to radiological assessment reflecting response to treatment would be of great utility for clinicians managing PM.

Due to the peculiar pattern of growth of PM, disease evaluation is quite difficult and remains an open challenge with a higher disagreement between radiologists than for other solid tumors. Modified RECIST criteria, defined since 2004, have enabled more accurate measurements of the pleural rind by implementing a standardized way to measure the tumor burden. Although this has brought an improvement in disease evaluation, high variability and imprecision in measurements remain and the application of mRECIST criteria still requires very expert and trained radiologists. As an alternative, tumor volume measurement on cross-sectional imaging has emerged as a potential tool with prognostic significance in PM [24,25]. In the last decade, this strategy has been evaluated as a potentially more reliable measure of chemotherapy response and predictor of outcomes in PM than RECIST [26]. Although promising, further validation of the role of tumor volume in clinical staging is required with large international studies before it can be incorporated into the clinical staging algorithm [27].

In our study to ensure the consistency in the evaluation of the mRECIST score we required all the CT scans to be reviewed by an expert radiologist blind to the clinical data and to the SMRP levels. Our results suggest that a doubling in SMRP level corresponded 
to an increase in mRECIST score of $16 \%$ and $18 \%$ in the AL and SP cohorts, respectively, and of approximately $20 \%$ when the two cohorts were analyzed together. According to mRECIST criteria, an increase of the mRECIST score of $20 \%$ is consistent with progressive disease. This finding, if confirmed in larger series, might provide new insight into the correlation between SMRP increase and disease extension changes.

The possibility of monitoring disease progression in PM patients is becoming increasingly important given the recent advances in systemic therapy, the promising results from second-line chemotherapy trials, as well as the availability of second-line experimental protocols.

One of the most controversial points among studies is the variation in the thresholds used to define a significant change in serum SMRP, as well as the appropriate sampling intervals during or after treatment. Our study suggests that the doubling of SMRP approximates a radiological progression disease based on mRECIST criteria. Guidelines on follow-up generically suggest that a 3/4-month interval is good practice, without indicating which kind of examinations should be performed. SMRP determinations could be readily used in the monitoring of patients in conjunction with, or even as an alternative to, radiological exams.

\section{Conclusions}

In conclusion, our exploratory proof of concept study has confirmed that SMRP could be a reliable biomarker for use in the monitoring of PM progression and suggests that the biomarker might assist in more accurate disease management and more efficient clinical decision-making. However, these data must be taken with caution and warrant further confirmatory cohort studies.

Author Contributions: Conceptualization, F.G. and V.F.; data curation, F.G., M.M., S.B. and M.P.P.; formal analysis, M.P.P. and V.F.; funding acquisition, M.P.P. and V.F.; investigation, F.U., P.F., M.C., R.G. and S.R.; methodology, F.G. and V.F.; project administration, F.G.; resources, F.U., A.V., A.M.D.A., S.D., M.L., S.B., S.R., U.G. and M.B.; supervision, V.F.; validation, M.C., R.G. and S.B.; visualization, M.L.; writing - original draft, F.G. and F.U.; writing—review \& editing, M.M., A.V., A.M.D.A., S.D., M.L., U.G. and M.B. All authors have read and agreed to the published version of the manuscript.

Funding: This work was partially funded by the Italian Ministry of Health, RC 2020 Project code M728A and RC 2021 Project code C728A, IRCCS Ospedale Policlinico San Martino Genova, to M.P.P. and V.F., respectively. Diagnostics and diagnostic instrumentation were provided free of charge by Fujirebio.

Institutional Review Board Statement: The study was conducted according to the guidelines of the Declaration of Helsinki and approved by the Ethics Committee of Azienda Ospedaliera SS Antonio e Biagio e Cesare Arrigo, Alessandria (authorization 1704; 27/10/2020) for the AL cohort and by the Ethics Committee of the Liguria Region (P.R. 207REG2014) for the SP cohort.

Informed Consent Statement: Informed consent was obtained from all subjects involved in the study.

Data Availability Statement: Patients' dataset (both cohorts) available.

Acknowledgments: The authors thank E. Battolla and the staff of the Laboratory of Clinical Pathology in which the analysis of mesothelin was performed, P.A. Canessa and the staff of the Pneumology Division for collecting serum at diagnosis, and P. Dessanti and the staff of the Clinical Pathology Division for histological diagnosis at the Azienda Sanitaria Locale n. 5, La Spezia, Italy.

Conflicts of Interest: F.G. reports, outside the submitted work, personal fees for an advisory role, speaker engagements and travel and accommodation expenses from Merck Sharp and Dohme, Novocure, Bristol Meyer Squibb, Boehringer Ingelheim, Pharmamar, and Novartis. The remaining authors declare no conflict of interest. The funders had no role in the design of the study, in the collection, analyses, or interpretation of data, in the writing of the manuscript, or in the decision to publish the results. 


\section{References}

1. Merritt, N.; Blewett, C.J.; Miller, J.D.; Bennett, W.F.; Young, J.E.; Urschel, J.D. Survival after conservative (palliative) management of pleural malignant mesothelioma. J. Surg. Oncol. 2001, 78, 171-174. [CrossRef] [PubMed]

2. Milano, M.T.; Zhang, H. Malignant pleural mesothelioma: A population-based study of survival. J. Thorac. Oncol. 2010, 5, 1841-1848. [CrossRef] [PubMed]

3. Vogelzang, N.J.; Rusthoven, J.J.; Symanowski, J.; Denham, C.; Kaukel, E.; Ruffie, P.; Gatzemeier, U.; Boyer, M.; Emri, S.; Manegold, C.; et al. Phase III study of pemetrexed in combination with cisplatin versus cisplatin alone in patients with malignant pleural mesothelioma. J. Clin. Oncol. 2003, 21, 2636-2644. [CrossRef]

4. $\quad$ Baas, P.; Scherpereel, A.; Nowak, A.K.; Fujimoto, N.; Peters, S.; Tsao, A.S.; Mansfield, A.S.; Popat, S.; Jahan, T.; Antonia, S.; et al. First-line nivolumab plus ipilimumab in unresectable malignant pleural mesothelioma (CheckMate 743): A multicentre, randomised, open-label, phase 3 trial. Lancet 2021, 397, 375-386. [CrossRef]

5. Zauderer, M.G.; Kass, S.L.; Woo, K.; Sima, C.S.; Ginsberg, M.S.; Krug, L.M. Vinorelbine and gemcitabine as second- or third-line therapy for malignant pleural mesothelioma. Lung Cancer 2014, 84, 271-274. [CrossRef]

6. Pagano, M.; Ceresoli, G.; Zucali, P.A.; Giulia, P.; Chiara, G.M.; Federica, G.; Marcello, T.; Parra, H.J.S.; Francesca, Z.; Federico, C.; et al. Randomized phase II study on gemcitabine with or without ramucirumab as second-line treatment for advanced malignant pleural mesothelioma (MPM): Results of Italian Rames Study. J. Clin. Oncol. 2020, 38, 9004. [CrossRef]

7. Fennell, D.; Ottensmeier, C.; Califano, R.; Hanna, G.G.; Ewings, S.; Kayleigh, H.; Wilding, S.; Danson, S.; Nye, M.; Steele, N. Nivolumab versus placebo in relapsed malignant mesothelioma: Preliminary results from the CONFIRM phase 3 trial. In Proceedings of the 2020 World Conference on Lung Cancer Singapore, Virtual, 28-31 January 2021.

8. Byrne, M.J.; Nowak, A.K. Modified RECIST criteria for assessment of response in malignant pleural mesothelioma. Ann. Oncol. 2004, 15, 257-260. [CrossRef]

9. Armato, S.G.; Nowak, A.K. Revised Modified Response Evaluation Criteria in Solid Tumors for Assessment of Response in Malignant Pleural Mesothelioma (Version 1.1). J. Thorac. Oncol. 2018, 13, 1012-1021. [CrossRef] [PubMed]

10. Robinson, B.W.; Creaney, J.; Lake, R.; Nowak, A.; Musk, A.W.; de Klerk, N.; Winzell, P.; Hellstrom, K.E.; Hellstrom, I. Mesothelinfamily proteins and diagnosis of mesothelioma. Lancet 2003, 362, 1612-1616. [CrossRef]

11. Hassan, R.; Laszik, Z.G.; Lerner, M.; Raffeld, M.; Postier, R.; Brackett, D. Mesothelin is overexpressed in pancreaticobiliary adenocarcinomas but not in normal pancreas and chronic pancreatitis. Am. J. Clin. Pathol. 2005, 124, 838-845. [CrossRef]

12. Ho, M.; Bera, T.K.; Willingham, M.C.; Onda, M.; Hassan, R.; FitzGerald, D.; Pastan, I. Mesothelin expression in human lung cancer. Clin. Cancer Res. 2007, 13, 1571-1575. [CrossRef] [PubMed]

13. Beyer, H.L.; Geschwindt, R.D.; Glover, C.L.; Tran, L.; Hellstrom, I.; Hellstrom, K.E.; Miller, M.C.; Verch, T.; Allard, W.J.; Pass, H.I.; et al. MESOMARK: A potential test for malignant pleural mesothelioma. Clin. Chem. 2007, 53, 666-672. [CrossRef] [PubMed]

14. Burt, B.M.; Lee, H.S.; Lenge De Rosen, V.; Hamaji, M.; Groth, S.S.; Wheeler, T.M.; Sugarbaker, D.J. Soluble Mesothelin-Related Peptides to Monitor Recurrence After Resection of Pleural Mesothelioma. Ann. Thorac. Surg. 2017, 104, 1679-1687. [CrossRef] [PubMed]

15. Hollevoet, K.; Van Cleemput, J.; Thimpont, J.; De Vuyst, P.; Bosquée, L.; Nackaerts, K.; Germonpré, P.; Vansteelandt, S.; Kishi, Y.; Delanghe, J.R.; et al. Serial measurements of mesothelioma serum biomarkers in asbestos-exposed individuals: A prospective longitudinal cohort study. J. Thorac. Oncol. 2011, 6, 889-895. [CrossRef] [PubMed]

16. Wheatley-Price, P.; Yang, B.; Patsios, D.; Patel, D.; Ma, C.; Xu, W.; Leighl, N.; Feld, R.; Cho, B.C.; O'Sullivan, B.; et al. Soluble mesothelin-related Peptide and osteopontin as markers of response in malignant mesothelioma. J. Clin. Oncol. 2010, 28, 3316-3322. [CrossRef] [PubMed]

17. Creaney, J.; Francis, R.J.; Dick, I.M.; Musk, A.W.; Robinson, B.W.; Byrne, M.J.; Nowak, A.K. Serum soluble mesothelin concentrations in malignant pleural mesothelioma: Relationship to tumor volume, clinical stage and changes in tumor burden. Clin. Cancer Res. 2011, 17, 1181-1189. [CrossRef]

18. Arnold, D.T.; De Fonseka, D.; Hamilton, F.W.; Rahman, N.M.; Maskell, N.A. Prognostication and monitoring of mesothelioma using biomarkers: A systematic review. Br. J. Cancer 2017, 116, 731-741. [CrossRef]

19. Fontana, V.; Vigani, A.; Pistillo, M.P.; Giannoni, U.; Rosemberg, I.; Canessa, P.A.; Berisso, G.; Ferro, P.; Franceschini, M.C.; Tonarelli, M.; et al. The Correlation of Serum Mesothelin Level With Pleural Thickness in Malignant Pleural Mesothelioma Makes it a Valuable Tool for Monitoring Tumor Progression. J. Thorac. Oncol. 2019, 14, e92-e94. [CrossRef]

20. Kindler, H.L.; Ismaila, N.; Armato, S.G.; Bueno, R.; Hesdorffer, M.; Jahan, T.; Jones, C.M.; Miettinen, M.; Pass, H.; Rimner, A.; et al. Treatment of Malignant Pleural Mesothelioma: American Society of Clinical Oncology Clinical Practice Guideline. J. Clin. Oncol. 2018, 36, 1343-1373. [CrossRef]

21. Fitzmaurice, G.M.; Laird, N.M.; Ware, J.H. Applied Longitudinal Analysis, 2nd ed.; Wiley: Hoboken, NJ, USA, 2011.

22. Grigoriu, B.D.; Chahine, B.; Vachani, A.; Gey, T.; Conti, M.; Sterman, D.H.; Marchandise, G.; Porte, H.; Albelda, S.M.; Scherpereel, A. Kinetics of soluble mesothelin in patients with malignant pleural mesothelioma during treatment. Am. J. Respir. Crit. Care Med. 2009, 179, 950-954. [CrossRef]

23. de Fonseka, D.; Arnold, D.T.; Stadon, L.; Morley, A.; Keenan, E.; Darby, M.; Armstrong, L.; Virgo, P.; Maskell, N.A. A prospective study to investigate the role of serial serum mesothelin in monitoring mesothelioma. BMC Cancer 2018, 18, 199. [CrossRef]

24. Pass, H.I.; Temeck, B.K.; Kranda, K.; Steinberg, S.M.; Feuerstein, I.R. Preoperative tumor volume is associated with outcome in malignant pleural mesothelioma. J. Thorac. Cardiovasc. Surg. 1998, 115, 310-317. [CrossRef] 
25. Gill, R.R.; Richards, W.G.; Yeap, B.Y.; Matsuoka, S.; Wolf, A.S.; Gerbaudo, V.H.; Bueno, R.; Sugarbaker, D.J.; Hatabu, H. Epithelial malignant pleural mesothelioma after extrapleural pneumonectomy: Stratification of survival with CT-derived tumor volume. AJR Am. J. Roentgenol. 2012, 198, 359-363. [CrossRef] [PubMed]

26. Frauenfelder, T.; Tutic, M.; Weder, W.; Götti, R.P.; Stahel, R.A.; Seifert, B.; Opitz, I. Volumetry: An alternative to assess therapy response for malignant pleural mesothelioma? Eur. Respir. J. 2011, 38, 162-168. [CrossRef] [PubMed]

27. Murphy, D.J.; Gill, R.R. Volumetric assessment in malignant pleural mesothelioma. Ann. Transl. Med. 2017, 5, 241. [CrossRef] [PubMed] 